\title{
Balanced and Approximate Zero-Variance Recursive Estimators for the Static Communication Network Reliability Problem
}

\author{
HECTOR CANCELA, Universidad de la República, Uruguay \\ MOHAMED EL KHADIRI, Saint-Nazaire Institute of Technology, France \\ GERARDO RUBINO and BRUNO TUFFIN, Inria Rennes Bretagne Atlantique, France
}

\begin{abstract}
Exact evaluation of static network reliability parameters belongs to the NP-hard family and Monte Carlo simulation is therefore a relevant tool to provide their estimations. The first goal of this paper is to review a Recursive Variance Reduction (RVR) estimator which approaches the unreliability by recursively reducing the graph from the random choice of the first working link on selected cuts. We show that the method does not verify the bounded relative error (BRE) property as reliability of individual links goes to one, i.e., that the estimator is not robust in general to high reliability of links. We then propose to use the decomposition ideas of the RVR estimator in conjunction with the Importance Sampling technique. Two new estimators are presented: the first one, called Balanced Recursive Decomposition estimator, chooses the first working link on cuts uniformly, while the second, called Zero-Variance Approximation Recursive Decomposition estimator, tries to mimic the estimator with variance zero for this technique. We show that in both cases BRE property is verified and, moreover, that a vanishing relative error (VRE) property can be obtained for the Zero-Variance Approximation RVR under specific sufficient conditions. A numerical illustration of the power of the methods is provided on several benchmark networks.
\end{abstract}

General Terms: Algorithms, Reliability, Performance

Additional Key Words and Phrases: aproximate zero variance, importance sampling, network reliability, Monte Carlo simulation, variance reduction

ACM Reference Format:

Hector Cancela, Mohamed El Khadiri, Gerardo Rubino and Bruno Tuffin, 2013. Balanced and Approximate Zero-Variance Recursive Estimators for the Static Communication Network Reliability Problem. ACM Trans. Model. Comput. Simul. V, N, Article (January YYYY), 18 pages.

DOI : http://dx.doi.org/10.1145/0000000.0000000

\section{INTRODUCTION}

The static reliability problem is used in the networking field, to know the probability that a group of nodes in the network can communicate. The communication network is represented by an undirected graph

$$
\mathcal{G}=(\mathcal{V}, \mathcal{E}, \mathcal{K})
$$

where

$-\mathcal{V}$ is the set of nodes,

$-\mathcal{E}=\{1, \ldots, m\}$ is the set of links connecting nodes, and

This work is supported by STIC-AMSUD Project AMMA

Author's addresses: Hector Cancela, Facultad de Ingeniería, Universidad de la República, Uruguay; Email: cancela@fing.edu.uy; Mohamed El Khadiri, Saint-Nazaire Institute of Technology, France, E-mail: Mohamed.El-Khadiri@univ-nantes.fr; Gerardo Rubino, Inria, France, E-mail: gerardo.rubino@inria.fr; Bruno Tuffin, Inria, France, E-mail: bruno.tuffin@inria.fr

Permission to make digital or hard copies of part or all of this work for personal or classroom use is granted without fee provided that copies are not made or distributed for profit or commercial advantage and that copies show this notice on the first page or initial screen of a display along with the full citation. Copyrights for components of this work owned by others than ACM must be honored. Abstracting with credit is permitted. To copy otherwise, to republish, to post on servers, to redistribute to lists, or to use any component of this work in other works requires prior specific permission and/or a fee. Permissions may be requested from Publications Dept., ACM, Inc., 2 Penn Plaza, Suite 701, New York, NY 10121-0701 USA, fax +1 (212) 869-0481, or permissions@acm.org.

(c) YYYY ACM 1049-3301/YYYY/01-ART $\$ 15.00$

DOI : http://dx.doi.org/10.1145/0000000.0000000 
$-\mathcal{K}$ is a subset of the node-set, called the terminal-set.

Nodes are assumed to be perfect, i.e., they do not fail, while links can fail, link $e \in \mathcal{E}$ failing with fixed (static) probability $q_{e}=1-r_{e}$. All failure events of individual links are assumed independent. The random vector-state, or configuration, of the network is given by the vector

$$
X=\left(X_{1}, \ldots, X_{m}\right)
$$

where for $1 \leq e \leq m, X_{e}$ is a Bernoulli random variable whose value is 1 if link $e$ is working, and 0 if it is failed. We aim at investigating the probability $r(\mathcal{G})$ that that all nodes in the terminal-set $\mathcal{K}$ are connected. We define a structure function $\Phi$ of $\{0,1\}^{m}$ in $\{0,1\}$ such that $\Phi(x)=1$ if all nodes in $\mathcal{K}$ are connected when the configuration is $x=\left(x_{1}, \ldots, x_{m}\right)$, and $\Phi(x)=0$ otherwise. We then have that $\Phi(X)$ is a Bernoulli random variable such that $\mathbb{E}[\Phi(X)]=r(\mathcal{G})$. In this paper we consider the computation of $r(\mathcal{G})$ or equivalently, the computation of the unreliability parameter

$$
q(\mathcal{G})=1-r(\mathcal{G})=\mathbb{E}[Y] \text { with } Y=1-\Phi(X) .
$$

The number of configurations is $2^{m}$, thus increasing exponentially with the number $m$ of links. Actually, the computation of $q(\mathcal{G})$ is known to be NP-hard [Ball 1986]. Exact combinatorial methods and bounding procedures, together with reduction techniques that allow to diminish the size of the models become quickly limited [Rubino 1998] as the size of the network increases. We can indeed observe that in communication networks, model sizes are often very large. Then Monte Carlo simulation methods [Asmussen and Glynn 2007] are alternatives leading to estimate $q(\mathcal{G})$ when $\mathcal{E}$ is of moderate to large size [Cancela et al. 2009].

Unfortunately, in the rare event case, the relative errors associated with the estimates produced by Monte-Carlo methods may be very large. A number of techniques have been published to deal with this problem, see for instance [Cancela and El Khadiri 1996; Elperin et al. 1991; Fishman 1986; Hui et al. 2005; Jun and Ross 1992; Kumamoto et al. 1980; Lomonosov 1994; Murray et al. 2013; Ross 1994; VillénAltamirano 2007]; an updated bibliography can be found in [Cancela et al. 2009]. In this paper, we focus on the Recursive Variance Reduction (RVR) estimator, one of most powerful methods available to solve this estimation problem [Cancela and El Khadiri 2003]. This technique recursively reduces the size of the considered network by considering a cut and looking at the first working link on this cut, sampled from the corresponding conditional distribution. The nodes connected by this link can then be merged while the previous non-working links are disregarded. A new cut is considered for the resulting reduced graph, and the process repeats recursively. Further improvement can be obtained by conditioning on both a cut and a path at the same time and looking for series-parallel reductions after the previously described step [Cancela and El Khadiri 1998; Cancela et al. 2012], but we will not use these variants here, to focus on the Importance Sampling effects.

The contributions of this paper are the following ones. We show that RVR does not verify the Bounded Relative Error (BRE) property and is not always asymptotically efficient as reliabilities of individual links increase. The BRE property is an important and standard qualitative measure of robustness in rare event simulation [Rubino and Tuffin 2009]. It states that the relative half-width of the confidence interval remains bounded whatever the rarity of the event. To obtain an improved method, we therefore propose to use the decomposition ideas of the RVR estimator, in conjunction with an Importance Sampling (IS) scheme, in order to ensure the BRE property. Two Importance Sampling strategies are considered. First, we propose to sample the first working link on a cut according to a uniform distribution, and show that this leads to the BRE 
property. Secondly, we exploit the zero-variance change of measure for proposing another way of sampling this link. This change-of measure is expressed in terms of the (exact) unreliability of the graph knowing the state of the links in the chosen cut. Of course, if we knew it, there would be no need to use simulation, but this provides an ideal expression and we can try to plug an approximation of those unreliabilities instead of the exact ones. The candidate we propose to use is the probability of a specific type of mincut (one maximizing the probability that all its components are down). Importance Sampling using the zero-variance approximation has recently been applied to this model [L'Ecuyer et al. 2011], dealing to an estimator for which the BRE property is verified, as well as the Vanishing Relative Error (VRE) property under supplementary conditions (this property corresponds to the case where the relative error goes to zero when the rarity of the event augments). The combination of IS with the RVR ideas we propose here is expected to yield an improved behavior in terms of reduced variance with respect to Importance Sampling, and in terms of better asymptotic properties with respect to RVR. We show that BRE is verified in general, that we get VRE under some (new) conditions, and provide numerical evidence about the improvement brought by the combination.

The paper is organized as follows. In Section 2 we recall the standard Monte Carlo method and the BRE property. The RVR method and the asymptotic analysis of its variance are the object of Section 3. In Section 4, we propose a new recursive sampling which leads to an estimator verifying BRE. Section 5 presents an Importance Sampling strategy that approaches the zero-variance change of measure when sampling the first working link on a cut. Numerical illustrations appear in Section 6. Some conclusions and possible improvements are given in Section 7.

\section{A REMINDER ON MONTE CARLO SIMULATION AND ROBUSTNESS PROPERTIES}

We first recall how Monte Carlo simulation works, when estimating the unreliability of a graph $(q(\mathcal{G})$ in Equation 1), or any other expectation, based on a sample mean.

For a fixed sample size $n$, the sample mean estimator of $q(\mathcal{G})$ is the average value

$$
\widehat{Y}_{n}=\frac{1}{n} \sum_{i=1}^{n} Y^{(i)}
$$

where $Y^{(i)}$ are i.i.d random variables following the law of any random variable $Y$ such that $\mathbb{E}[Y]=q(\mathcal{G}))$.

The corresponding variance is $\operatorname{Var}\left[\widehat{Y}_{n}\right]=\operatorname{Var}[Y] / n$, estimated for example by $\sum_{i=1}^{n}\left(Y^{(i)}-\widehat{Y}\right)^{2} /(n-1)$. The Central Limit Theorem then provides, for $n$ large enough, a confidence interval at level $\alpha$ given by

$$
\left(\widehat{Y}_{n}-c_{\alpha} \sqrt{\operatorname{Var}\left[\widehat{Y}_{n}\right]}, \widehat{Y}_{n}+c_{\alpha} \sqrt{\operatorname{Var}\left[\widehat{Y}_{n}\right]}\right),
$$

where $c_{\alpha}=\phi^{-1}((1+\alpha) / 2)$ and $\phi$ is the cumulative distribution function of the standard Normal law.

The relative error produced by the estimator is defined as the relative half-size of the confidence interval, given by

$$
R E=c_{\alpha} \frac{\sqrt{\operatorname{Var}\left[\widehat{Y}_{n}\right]}}{\mathbb{E}[Y]} .
$$

In the particular case of network reliability, the standard Monte Carlo simulation (SMC, or also sometimes called crude MC) defines the estimator of $q(\mathcal{G})$ as the sample 
average $\widehat{Y}_{n}=\frac{1}{n} \sum_{i=1}^{n} Y^{(i)}$ where $Y^{(i)}=1-\Phi\left(X^{(i)}\right)$ and the $X^{(i)} \mathrm{s}, X^{(i)}=\left(X_{1}^{(i)}, \ldots, X_{m}^{(i)}\right)$, $1 \leq i \leq n$, are $n$ independent trials of $X$. The corresponding variance is $\operatorname{Var}\left[\widehat{Y}_{n}\right]=$ $r(\overline{\mathcal{G}}) q(\mathcal{G}) / n=q(\mathcal{G})(1-q(\mathcal{G})) / n$,

There are unfortunately difficulties when applying the SMC estimator for evaluating highly reliable networks. First, it is unlikely that one of the $Y^{(i)}$ is equal to one, i.e., that the rare event is reached, unless the sample size $n$ is large (it requires in average $n=1 / q(\mathcal{G})$ to observe the event once). Also, even if $n$ is large enough, the relative error, that is the relative half-size of the confidence interval, verifies

$$
R E=c_{\alpha} \frac{\sqrt{q(\mathcal{G}) r(\mathcal{G})}}{q(\mathcal{G}) \sqrt{n-1}} \rightarrow \infty \text { as } q(\mathcal{G}) \rightarrow 0 .
$$

This property means that, if we fix in advance a specified relative error to reach, the sample size needed to attain it must increase (up to infinity) when $q(\mathcal{G}) \rightarrow 0$.

Variance reduction techniques can be applied to get a more efficient estimator $\widehat{Y}_{n}^{\prime}$ of $\mathbb{E}[Y]$. Several methods have been proposed for the network unreliability estimation (see [Cancela et al. 2009] for a survey). The method we are going to use in conjunction with RVR is the Importance Sampling (IS) technique [Asmussen and Glynn 2007; Rubino and Tuffin 2009]. Shortly, this technique consists, when computing the expectation $\mathbb{E}[Y]$ of a r.v. $Y$ under probability distribution $\mathbb{P}$, to use instead a distribution $\widetilde{\mathbb{P}}$ such that $d \widetilde{\mathbb{P}}>0$ whenever $Y d \mathbb{P}>0$. In that case

$$
\mathbb{E}[Y]=\int Y(\omega) d \mathbb{P}(\omega)=\int Y(\omega) \frac{d \mathbb{P}(\omega)}{d \widetilde{\mathbb{P}}(\omega)} d \widetilde{\mathbb{P}}(\omega)=\widetilde{\mathbb{E}}[Y L]
$$

where $\widetilde{\mathbb{E}}$ is the expectation under probability $\widetilde{\mathbb{P}}$ and $L=d \mathbb{P} / d \widetilde{\mathbb{P}}$ is the likelihood ratio. We can then compute the same value by using a weighted (by $L$ ) version of the random variable $Y$, under another probability distribution. By a proper choice of $\widetilde{\mathbb{P}}$, the variance can be reduced.

Some properties a rare event estimator should enjoy are the so-called Bounded Relative Error (BRE) and the Vanishing Relative Error (VRE) ones [Glynn et al. 2009; L'Ecuyer et al. 2010].

Definition 2.1. An estimator $\widehat{Y}_{n}^{\prime}$ of $\mathbb{E}[Y]$ verifies Bounded Relative Error (BRE) if the half-width relative value of the confidence interval remains bounded as the event becomes rarer. In other words, the sample size required to get a given relative error is not sensitive to the rarity of the event. Formally, we have BRE if $c_{\alpha} \sqrt{\operatorname{Var}\left[\widehat{Y}_{n}^{\prime}\right]} / \mathbb{E}[Y]$ remains bounded as $\mathbb{E}[Y] \rightarrow 0$.

Definition 2.2. The estimator is said to verify the Vanishing Relative Error (VRE) property if $\sqrt{\operatorname{Var}\left[\widehat{Y}_{n}^{\prime}\right]} / \mathbb{E}[Y] \rightarrow 0$ as $\mathbb{E}[Y] \rightarrow 0$. In words, the relative error decreases to zero with the probability of the event, the relative precision improves with rarity.

Remark 2.3. As

$$
\operatorname{Var}\left[\widehat{Y}_{n}^{\prime}\right]=\mathbb{E}\left[\left(\widehat{Y}_{n}^{\prime}\right)^{2}\right]-\left(\mathbb{E}\left[\widehat{Y}_{n}^{\prime}\right]\right)^{2}=\mathbb{E}\left[\left(\widehat{Y}_{n}^{\prime}\right)^{2}\right]-(\mathbb{E}[Y])^{2},
$$

the estimator $\widehat{Y}_{n}^{\prime}$ has the BRE property if and only if

$$
\mathbb{E}\left[\left(\widehat{Y}_{n}^{\prime}\right)^{2}\right] /(\mathbb{E}[Y])^{2} \text { remains bounded as } \mathbb{E}[Y] \rightarrow 0 .
$$

In the next section, we are going to focus on the Recursive Variance Reduction (RVR) estimator, one of the most successful methods in the area. But we want to analyze 
its efficiency as the unreliability of individual links $q_{e}$ go to zero $\forall e \in \mathcal{E}$. In order to formally analyze this, we introduce a rarity parameter $\epsilon \ll 1$ such that, $\forall e \in \mathcal{E}$,

$$
q_{e}=a_{e} \epsilon^{b_{e}}
$$

with $a_{e}, b_{e}$ positive constants [Heidelberger et al. 1994]. Therefore $q_{e} \rightarrow 0$ as $\epsilon \rightarrow 0$.

As general notation, given two functions $f$ and $g$, we will say that $f$ is in $O(g(\epsilon))$ (resp., in $\Theta(g(\epsilon))$ ) if $f(\epsilon) \leq c_{2} g(\epsilon)$ (resp. $c_{1} g(\epsilon) \leq f(\epsilon) \leq c_{2} g(\epsilon)$ ) when $\epsilon \rightarrow 0$, for some positive constants $c_{1}$ and $c_{2}$. We will also say that $f$ is in $o(g(\epsilon))$ if $f(\epsilon) / g(\epsilon) \rightarrow 0$ when $\epsilon \rightarrow 0$.

Using this notation, and under the previous assumption, due to the finite state space, it is easy to see that there exists a constant $d>0$ such that

$$
q(\mathcal{G})=\Theta\left(\epsilon^{d}\right),
$$

as a sum of probabilities of configurations $x$ such that $\Phi(x)=0$, which are all polynomials in $\epsilon$. As a consequence $q(\mathcal{G})=\mathbb{E}[Y] \rightarrow 0$ too as $\epsilon \rightarrow 0$.

\section{RECURSIVE VARIANCE REDUCTION (RVR) METHOD AND ASYMPTOTIC ANALYSIS OF ITS VARIANCE}

To define the RVR estimator [Cancela and El Khadiri 2003], we first need to recall some graph theory concepts. Recall that a cut or $\mathcal{K}$-cut in the graph $\mathcal{G}$ is a set of links such that if we remove them from $\mathcal{G}$, the nodes in $\mathcal{K}$ are not in the same connected component of the resulting graph. A mincut (minimal cut) is a cut that contains no other cut than itself. The probability $q_{\mathcal{C}}$ of a mincut $\mathcal{C}$ is defined as the probability that all its links fail; and it can be computed by $q_{\mathcal{C}}=\prod_{e \in \mathcal{C}} q_{e}$. For any $\mathcal{K}$-cut $\mathcal{C}, q_{\mathcal{C}} \leq q(\mathcal{G})=$ $\Theta\left(\epsilon^{d}\right)$. Then, for any $\mathcal{C}, q_{\mathcal{C}}=\Theta\left(\epsilon^{c}\right)$, with $c \geq d$; and there will always be at least one $\mathcal{K}$-cut $\mathcal{C}$, such that $q_{\mathcal{C}}=\Theta\left(\epsilon^{d}\right)$.

Basically, the idea behind RVR is first to select a $\mathcal{K}$-cut $C$, whose elements are indexed in some arbitrary order, say $1,2, \ldots,|\mathcal{C}|$. The method consists then in writing the unreliability of $\mathcal{G}$ conditioning first on the fact that all the links in $\mathcal{C}$ are failed or not, and then, in the negative case, conditioning again with respect to the index of the first working link in $\mathcal{C}$ (given that there is at least one such link). The procedure will sample that link, obtaining some index value $J \in \mathcal{C}$, will remove links 1 to $J-1$ from $\mathcal{G}$, will merge the extremities of link $J$, and then, it will be applied again (recursively) on the resulting graph.

Formally, let us fix a $\mathcal{K}$-cut $\mathcal{C}$ of $\mathcal{G}$ and let us denote by $q_{\mathcal{C}}=\prod_{e \in \mathcal{C}} q_{e}$ the probability that all links in $\mathcal{C}$ are failed. Let $B_{j}$ be the event "the $j-1$ first links of $\mathcal{C}$ are down and the $j$ th is up", $j=1, \ldots,|\mathcal{C}|$, with probability $\mathbb{P}\left[B_{j}\right]=r_{j} \prod_{k=1}^{j-1} q_{k}$.

The RVR estimator proposed in [Cancela and El Khadiri 2003] is a sample mean based on $n$ independent trials of the following recursively defined random variable:

$$
Y_{R V R}=q_{\mathcal{C}}+\left(1-q_{\mathcal{C}}\right) \sum_{j=1}^{|\mathcal{C}|} \mathbf{1}(J=j) Y_{R V R}\left(\mathcal{G}_{j}\right)
$$

where

$-\mathbf{1}(B)$ is the r.v. "indicator function of event $B$ ";

$-J$ is a r.v. defined on the indexes of the links in $\mathcal{C}$, with

$$
\mathbb{P}[J=j]=\mathbb{P}\left[B_{j} \mid \text { at least one link of } \mathcal{C} \text { is working }\right]=\frac{\mathbb{P}\left(B_{j}\right)}{1-q_{\mathcal{C}}}=p_{j} ;
$$

$-\mathcal{G}_{j}$ is the graph obtained from $\mathcal{G}$ after removing the $j-1$ first links of $\mathcal{C}$ and merging the extremities of the $j$ th. 


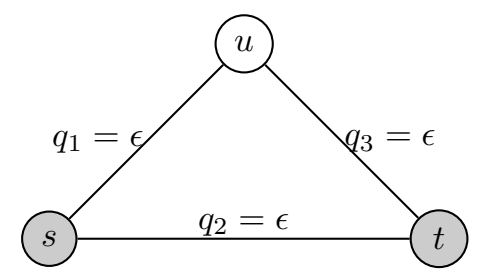

Fig. 1. Simple graph topology for which RVR does not verify BRE

¿From an algorithmic point of view, the different steps are:

- Select a $\mathcal{K}$-cut, order the edges, compute $q_{\mathcal{C}}$ and the $p_{j}$ s.

- Pick an edge $J$ at random in $C$ with probability distribution $\left(p_{j}\right)_{j=1, \cdots,|\mathcal{C}|}$, representing the first working edge on the $\mathcal{K}$-cut. The $J-1$ first edges are therefore considered failed, while the state of the other ones are unknown.

- Call $\mathcal{G}_{J}$ the graph obtained from $\mathcal{G}$ by deleting the first $J-1$ edges of $\mathcal{C}$ and by contracting the $J$ th one.

- The value $y_{R V R}$ returned by the RVR estimator of $q(\mathcal{G})$, the unreliability of $\mathcal{G}$, is recursively defined as

$$
y_{R V R}(\mathcal{G})=q_{\mathcal{C}}+\left(1-q_{\mathcal{C}}\right) y_{R V R}\left(\mathcal{G}_{J}\right)
$$

up to a graph with terminal nodes not connected (returning 1 ), or connected (returning 0 ).

It is shown in [Cancela and El Khadiri 1995] that $\mathbb{E}\left[Y_{R V R}(\mathcal{G})\right]=q(\mathcal{G})$ (i.e., it is unbiased) and, after some algebra, it can be checked that

$$
\mathbb{E}\left[Y_{R V R}(\mathcal{G})\right]=q_{\mathcal{C}}+\sum_{j=1}^{|\mathcal{C}|} \mathbb{P}\left[B_{j}\right] \mathbb{E}\left[Y_{R V R}\left(\mathcal{G}_{j}\right)\right]
$$

and

$$
\mathbb{E}\left[Y_{R V R}^{2}(\mathcal{G})\right]=q_{\mathcal{C}}^{2}+2 q_{\mathcal{C}}\left(\sum_{j=1}^{|\mathcal{C}|} \mathbb{P}\left[B_{j}\right] \mathbb{E}\left[Y_{R V R}\left(\mathcal{G}_{j}\right)\right]\right)+\left(1-q_{\mathcal{C}}\right)\left(\sum_{j=1}^{|\mathcal{C}|} \mathbb{P}\left[B_{j}\right] \mathbb{E}\left[Y_{R V R}^{2}\left(\mathcal{G}_{j}\right)\right]\right)(9)
$$

The RVR has been empirically illustrated to be an efficient technique on many different topologies, giving an unbiased estimator of the unreliability, with an important variance reduction with respect to standard Monte Carlo SMC. We point out now its limits as the unreliability of individual links goes to zero.

Proposition 3.1. The RVR estimator does not verify the Bounded Relative Error property in general as the unreliability of individual links goes to zero.

Proof. Consider the simple graph $\mathcal{G}$ of Figure 1, where we want to compute the probability $q(\mathcal{G})$ that the two (grey) nodes $s$ and $t$ are not connected. The unreliability of link $e$ is $q_{e}=\epsilon$.

Let us choose the cut with the two links starting from node $s$ ordered such as $\{s, t\}$ is the first link and $\{s, u\}$ is the second one. We then have $q_{\mathcal{C}}=\epsilon^{2}$. The reduced graphs are represented in Figure 2. In that figure, $\mathcal{G}_{1}$ is the graph deduced from $\mathcal{G}$ by fixing $\{s, t\}$ at the working state. Nodes $s$ and $t$ are then merged. For graph $\mathcal{G}_{2},\{s, t\}$ is failed and thus removed, while $\{s, u\}$ is working, so that the two nodes are merged.

For the graph $\mathcal{G}_{1}$, as $s$ and $t$ are merged, we obtain $Y_{R V R}\left(\mathcal{G}_{1}\right)=Y_{R V R}^{2}\left(\mathcal{G}_{1}\right)=0$. For $\mathcal{G}_{2}$, the remaining link $\{s, t\}$ has unreliability $\epsilon$. Then RVR is again used with the (single) 

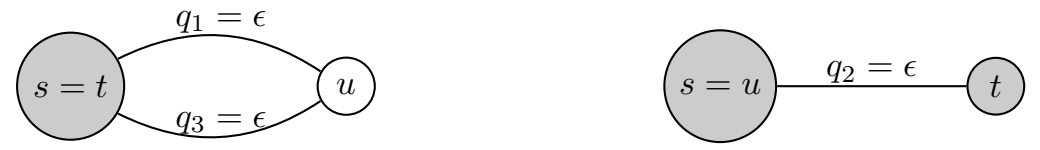

Fig. 2. Reduced graphs when applying RVR to the example of Figure 1, taking links in order $\{s, t\},\{s, u\}$. Left: $\mathcal{G}_{1} ;$ right: $\mathcal{G}_{2}$.
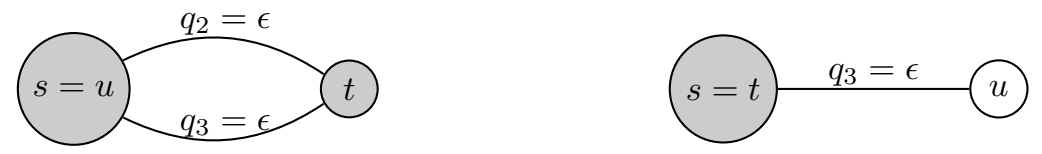

Fig. 3. Reduced graphs when applying RVR to the example of Figure 1, taking links in order $\{s, u\},\{s, t\}$. Left: $\mathcal{G}_{1}$; right: $\mathcal{G}_{2}$.

cut containing the link $\{s, t\}$, leading to $Y_{R V R}\left(\mathcal{G}_{2}\right)=\epsilon$ and $Y_{R V R}^{2}\left(\mathcal{G}_{2}\right)=\epsilon^{2}$. Based on these results, equality (8) and equality (9), we obtain

$$
\begin{aligned}
\mathbb{E}\left[Y_{R V R}(\mathcal{G})\right] & =\epsilon^{2}+(1-\epsilon) \mathbb{E}\left[Y_{R V R}\left(\mathcal{G}_{1}\right)\right]+\epsilon(1-\epsilon) \mathbb{E}\left[Y_{R V R}\left(\mathcal{G}_{2}\right)\right] \\
& =\epsilon^{2}+\epsilon(1-\epsilon) \mathbb{E}\left[Y_{R V R}\left(\mathcal{G}_{2}\right)\right]=\epsilon^{2}+\epsilon(1-\epsilon) \epsilon^{2}=2 \epsilon^{2}-\epsilon^{3}
\end{aligned}
$$

and

$$
\begin{aligned}
\mathbb{E}\left[Y_{R V R}^{2}(\mathcal{G})\right]= & \epsilon^{4}+2 \epsilon^{2}\left((1-\epsilon) \mathbb{E}\left[Y_{R V R}\left(\mathcal{G}_{1}\right)\right]+\epsilon(1-\epsilon) \mathbb{E}\left[Y_{R V R}\left(\mathcal{G}_{2}\right)\right]\right) \\
& +\left(1-\epsilon^{2}\right)\left((1-\epsilon) \mathbb{E}\left[Y_{R V R}^{2}\left(\mathcal{G}_{1}\right)\right]+\epsilon(1-\epsilon) \mathbb{E}\left[Y_{R V R}^{2}\left(\mathcal{G}_{2}\right)\right]\right) \\
= & \epsilon^{4}+2 \epsilon^{2}\left(\epsilon(1-\epsilon) \mathbb{E}\left[Y_{R V R}\left(\mathcal{G}_{2}\right)\right]\right)+\left(1-\epsilon^{2}\right)\left(\epsilon(1-\epsilon) \mathbb{E}\left[Y_{R V R}^{2}\left(\mathcal{G}_{2}\right)\right]\right) \\
= & \epsilon^{4}+2 \epsilon^{2}(\epsilon(1-\epsilon) \epsilon)+\left(1-\epsilon^{2}\right)\left(\epsilon(1-\epsilon) \epsilon^{2}\right)=\epsilon^{3}+2 \epsilon^{4}-3 \epsilon^{5}+\epsilon^{6}
\end{aligned}
$$

As a consequence, $\mathbb{E}\left[Y_{R V R}^{2}(\mathcal{G})\right] /\left(\mathbb{E}\left[Y_{R V R}(\mathcal{G})\right]\right)^{2}=\Theta\left(\epsilon^{-1}\right) \rightarrow \infty$ as $\epsilon \rightarrow 0$, and the BRE property is not verified from Remark 2.3.

Remark 3.2. Suppose now that the first link of the considered cut is $\{s, u\}$ and the second is $\{s, t\}$. Then $q_{\mathcal{C}}=\epsilon^{2}$ but $\mathcal{G}_{1}$ and $\mathcal{G}_{2}$ differ from the preceding case. They are represented in Figure 3: $\mathcal{G}_{1}$ is the graph deduced from $\mathcal{G}$ by fixing $\{s, u\}$ as working and $\mathcal{G}_{2}$ by fixing $\{s, u\}$ as failed and $\{s, t\}$ working.

Then for $\mathcal{G}_{2}$ we have $Y_{R V R}\left(\mathcal{G}_{2}\right)=Y_{R V R}^{2}\left(\mathcal{G}_{2}\right)=0$ and for $\mathcal{G}_{1}$, RVR is again used with the (single) cut containing the two links $\{s, t\}$ and $\{u, t\}$ (remember that $s$ and $u$ are now merged in $\mathcal{G}_{1}$, so that these are two parallel links from the single node $s=u$ to the node $t$ ), leading to $Y_{R V R}\left(\mathcal{G}_{1}\right)=\epsilon^{2}$ and $Y_{R V R}^{2}\left(\mathcal{G}_{1}\right)=\epsilon^{4}$. Based on these results, we obtain from (8) and (9),

$$
\begin{aligned}
\mathbb{E}\left[Y_{R V R}(\mathcal{G})\right] & =\epsilon^{2}+(1-\epsilon) \mathbb{E}\left[Y_{R V R}\left(\mathcal{G}_{1}\right)\right]+\epsilon(1-\epsilon) \mathbb{E}\left[Y_{R V R}\left(\mathcal{G}_{2}\right)\right] \\
& =\epsilon^{2}+(1-\epsilon) \mathbb{E}\left[Y_{R V R}\left(\mathcal{G}_{1}\right)\right]=\epsilon^{2}+(1-\epsilon) \epsilon^{2}=2 \epsilon^{2}-\epsilon^{3}
\end{aligned}
$$

and

$$
\begin{aligned}
\mathbb{E}\left[Y_{R V R}^{2}(\mathcal{G})\right]= & \epsilon^{4}+2 \epsilon^{2}\left((1-\epsilon) \mathbb{E}\left[Y_{R V R}\left(\mathcal{G}_{1}\right)\right]+\epsilon(1-\epsilon) \mathbb{E}\left[Y_{R V R}\left(\mathcal{G}_{2}\right)\right]\right) \\
& +\left(1-\epsilon^{2}\right)\left((1-\epsilon) \mathbb{E}\left[Y_{R V R}^{2}\left(\mathcal{G}_{1}\right)\right]+\epsilon(1-\epsilon) \mathbb{E}\left[Y_{R V R}^{2}\left(\mathcal{G}_{2}\right)\right]\right) \\
= & \epsilon^{4}+2 \epsilon^{2}\left((1-\epsilon) \mathbb{E}\left[Y_{R V R}\left(\mathcal{G}_{1}\right)\right]\right)+\left(1-\epsilon^{2}\right)\left((1-\epsilon) \mathbb{E}\left[Y_{R V R}^{2}\left(\mathcal{G}_{1}\right)\right]\right) \\
= & \epsilon^{4}+2 \epsilon^{2}\left((1-\epsilon) \epsilon^{2}\right)+\left(1-\epsilon^{2}\right)\left((1-\epsilon) \epsilon^{4}\right)=4 \epsilon^{4}-3 \epsilon^{5}-\epsilon^{6}+2 \epsilon^{7} .
\end{aligned}
$$

ACM Transactions on Modeling and Computer Simulation, Vol. V, No. N, Article, Publication date: January YYYY. 
As a consequence, $\mathbb{E}\left[Y_{R V R}^{2}(\mathcal{G})\right] /\left(\mathbb{E}\left[Y_{R V R}(\mathcal{G})\right]\right)^{2}=\Theta(1)$ as $\epsilon \rightarrow 0$. BRE prop-

erty is thus obtained. Moreover, in this case we have that $\sqrt{\operatorname{Var}\left[\widehat{Y}_{n}^{\prime}\right]} / \mathbb{E}[Y]=$ $\sqrt{4 \epsilon^{4}-3 \epsilon^{5}-\epsilon^{6}+2 \epsilon^{7}-\left(2 \epsilon^{2}-\epsilon^{3}\right)^{2}} /\left(2 \epsilon^{2}-\epsilon^{3}\right) \rightarrow 0$ as $\epsilon \rightarrow 0$, leading to verification of the VRE property. The order of links therefore plays an important role on the resulting relative error.

Remark 3.3. To better understand why BRE can be observed or not, consider again the last term of (9). One can check that the second moment is expressed in terms of the (sum of) second moments for subgraphs $\mathcal{G}_{j}$ multiplied by the probability of $B_{j}$, while the square of the expectation is the square of the sum of expectations for subgraphs $\mathcal{G}_{j}$ multiplied by the probability of $B_{j}$ as described in (8). Remember that $q(\mathcal{G})=\Theta\left(\epsilon^{d}\right)$. In order to get the BRE property, we necessarily need that the exponent of $\epsilon$ in $\mathbb{P}\left[B_{j}\right] \mathbb{E}\left[Y_{R V R}^{2}\left(\mathcal{G}_{j}\right)\right]$ be at least $2 d$. For the indices $j$ such that $\mathbb{P}\left[B_{j}\right] \mathbb{E}\left[Y_{R V R}\left(\mathcal{G}_{j}\right)\right]=\Theta(q(\mathcal{G}))=\Theta\left(\epsilon^{d}\right)$, this necessarily means that $\mathbb{P}\left[B_{j}\right]=\Theta(1)$, i.e., that sampling $B_{j}$ is not a rare event because its probability does not go to 0 with $\epsilon$. We will check these conditions in the example presented in Figure 1: in this case, $q(\mathcal{G})=\Theta\left(\epsilon^{2}\right)$, that is $d=2$. When looking at $\{s, t\}$ first and then $\{s, u\}$, the relevant $j$ is $j=2$, for which we have $\mathbb{P}\left[B_{2}\right]=\epsilon(1-\epsilon)$ and $\mathbb{E}\left[Y_{R V R}^{2}\left(\mathcal{G}_{2}\right)\right]=\epsilon^{2}$, hence the condition is not verified, and we don't have BRE. On the other hand, when looking at $\{s, u\}$ first and then $\{s, t\}$ we have that the term corresponding to $j=1$ verifies $\mathbb{P}\left[B_{1}\right] \mathbb{E}\left[Y_{R V R}^{2}\left(\mathcal{G}_{1}\right)\right]=\Theta\left(\epsilon^{4}\right)$, which was the necessary condition; the term corresponding to $j=2$ has $\mathbb{E}\left[Y_{R V R}\left(\mathcal{G}_{2}\right)\right]=\mathbb{E}\left[Y_{R V R}^{2}\left(\mathcal{G}_{2}\right)\right]=0$, so it does not contribute neither to the estimation nor to the variance.

This remark is the starting point for designing a new method based on the ideas of RVR, but which always yields the BRE property.

\section{BALANCED RECURSIVE DECOMPOSITION ESTIMATOR}

Consider now the case where we combine the recursive decomposition ideas of RVR with IS. IS is used by changing the probabilities $\left(p_{j}\right)$ for choosing the first line up in the cut. More formally, if you consider definition (7), we substitute the distribution of $J$ by a new sequence $\left(\widetilde{p}_{j}\right)_{j=1, \cdots,|\mathcal{C}|}$.

We recall here that we built a partition by assigning to the events $B_{j}$, for $1 \leq j \leq|\mathcal{C}|$, the conditional probabilities

$$
p_{j}=\mathbb{P}\left(B_{j} \mid A\right)=\frac{\mathbb{P}\left[B_{j}\right]}{1-q_{\mathcal{C}}}
$$

where $A$ is the event "at least one link in cut $\mathcal{C}$ is up". Denote by $B_{j}^{\prime}$ as the same event $B_{j}$ but on the probability space defined conditionally to $A$. In this probability space, $B_{j}^{\prime}$ has probability $p_{j}$. The RVR estimator can be expressed as

$$
Y_{R V R}=q_{\mathcal{C}}+\left(1-q_{\mathcal{C}}\right) \sum_{j=1}^{|\mathcal{C}|} \mathbf{1}_{B_{j}^{\prime}} Y_{R V R}\left(\mathcal{G}_{j}\right) .
$$

Instead of using the probabilities $p_{j}$, one can apply IS by making use of another probability law $\widetilde{\mathbb{P}}$ and expectation $\widetilde{\mathbb{E}}$, thanks to new probabilities $\widetilde{p}_{j}$, so that a general IS new estimator can be defined by

$$
\widetilde{Y}_{I S}(\mathcal{G})=q_{\mathcal{C}}+\left(1-q_{\mathcal{C}}\right) \sum_{j=1}^{|\mathcal{C}|} \mathbf{1}_{B_{j}^{\prime}(\mathcal{G})} \frac{p_{j}}{\widetilde{p}_{j}} Y_{I S}\left(\mathcal{G}_{j}\right) .
$$


We have added the likelihood ratio $p_{j} / \widetilde{p}_{j}$ in the estimator to keep it unbiased.

We first consider a uniform distribution $\widetilde{p}_{j}=1 /|\mathcal{C}|$, for sampling $B_{j}^{\prime}$, as a particular case of this IS estimator, inspired by the related work on Highly Reliable Markovian Systems [Nakayama 1996]. This balances the choice of the first link up, hence the name Balanced Recursive Decomposition (BRD). The resulting estimator is

$$
Y_{B R D}(\mathcal{G})=q_{\mathcal{C}}+|\mathcal{C}| \sum_{j=1}^{|\mathcal{C}|} \mathbf{1}_{B_{j}^{\prime}(\mathcal{G})} \mathbb{P}\left[B_{j}(\mathcal{G})\right] Y_{B R D}\left(\mathcal{G}_{j}\right) .
$$

This is an unbiased estimator of the unreliability, which moreover verifies the desired property:

Proposition 4.1. The BRD estimator verifies the Bounded Relative Error property.

PROOF. First notice that

$$
\begin{aligned}
\widetilde{\mathbb{E}}\left[Y_{B R D}^{2}(\mathcal{G})\right]= & q_{\mathcal{C}}^{2}+2 q_{\mathcal{C}}\left(\sum_{j=1}^{|\mathcal{C}|} \mathbb{P}\left[B_{j}(\mathcal{G})\right] \widetilde{\mathbb{E}}\left[Y_{B R D}\left(\mathcal{G}_{j}\right)\right]\right) \\
& +|\mathcal{C}|\left(\sum_{j=1}^{|\mathcal{C}|}\left(\mathbb{P}\left[B_{j}(\mathcal{G})\right]\right)^{2} \widetilde{\mathbb{E}}\left[Y_{B R D}^{2}\left(\mathcal{G}_{j}\right)\right]\right) .
\end{aligned}
$$

The proof then proceeds by induction. Let $c, d, f_{j}$ and $d_{j}$ be constants respectively such that $q_{\mathcal{C}}=\Theta\left(\epsilon^{c}\right), \widetilde{\mathbb{E}}\left[Y_{B R D}(\mathcal{G})\right]=\Theta\left(\epsilon^{d}\right), \mathbb{P}\left[B_{j}(\mathcal{G})\right]=\Theta\left(\epsilon^{f_{j}}\right)$ and $\widetilde{\mathbb{E}}\left[Y_{B R D}\left(\mathcal{G}_{j}\right)\right]=\Theta\left(\epsilon^{d_{j}}\right)$. Those constants exist because the expectations are finite sums of polynomials in $\epsilon$ ¿From $(11) d=\min _{j}\left(c, f_{j}+d_{j}\right)$. If by the assumption of the induction $\forall j, \widetilde{\mathbb{E}}\left[Y_{B R D}^{2}\left(\mathcal{G}_{j}\right)\right]=$ $\Theta\left(\left(\widetilde{\mathbb{E}}\left[Y_{B R D}\left(\mathcal{G}_{j}\right)\right]\right)^{2}\right)$, we have from (12), $\widetilde{\mathbb{E}}\left[Y_{B R D}^{2}(\mathcal{G})\right]=\Theta\left(\epsilon^{\min _{j}\left(2 c, c+f_{j}+d_{j}, 2 f_{j}+2 d_{j}\right)}\right)$ thus

$$
\widetilde{\mathbb{E}}\left[Y_{B R D}^{2}(\mathcal{G})\right]=\Theta\left(\epsilon^{2 d}\right)=\Theta\left(\left(\widetilde{\mathbb{E}}\left[Y_{B R D}(\mathcal{G})\right]\right)^{2}\right) .
$$

For the simplest topologies (the ones working as soon as a single link is working), the property is also verified, which completes the proof.

Remark 4.2. The intuition behind BRD is to make sure that the probability of each event $B_{j}^{\prime}$ is $\Theta(1)$ as highlighted in Remark 3.3, so that no event is rare under IS. As a consequence the probability $\mathbb{P}\left[B_{j}(\mathcal{G})\right]$ is squared in the likelihood ratio (which was not the case for RVR), so that BRE can be obtained now. Note that any choice of distribution, provided that the probability of each event $B_{j}^{\prime}$ is $\Theta(1)$, can be taken in place of the uniform one and yields the same result.

Remark 4.3. It is interesting to note that it is possible to build network topologies where for certain link unreliability values SMC has lower variance than BRD (this will be highlighted within our numerical experiments). Thus, the BRD estimator does not guarantee variance reduction in all contexts although in the rare event context it will be much more precise than standard Monte Carlo (as the former verifies the BRE property, and the latter does not).

\section{ZERO-VARIANCE RECURSIVE DECOMPOSITION ESTIMATOR}

Instead of sampling the first working component in $\mathcal{C}$ by a uniform distribution in BRD, we can look at an importance sampling strategy which would lead to variance zero. Zero-variance approximation is indeed a promising area in Monte Carlo simulation 
[L'Ecuyer and Tuffin 2008]. The idea is, somewhat similarly to [L'Ecuyer et al. 2011], to select $B_{j}^{\prime}$ in the estimator (10), with probability

$$
\widetilde{p}_{j}=\widetilde{\mathbb{P}}\left[B_{j}^{\prime}\right]=\frac{\mathbb{P}\left[B_{j}\right] q\left(\mathcal{G}_{j}\right)}{\sum_{k=1}^{|\mathcal{C}|} \mathbb{P}\left[B_{k}\right] q\left(\mathcal{G}_{k}\right)} .
$$

The resulting estimator is expressed as:

$$
\begin{aligned}
Y_{Z V R D} & =q_{\mathcal{C}}+\left(1-q_{\mathcal{C}}\right) \sum_{j=1}^{|\mathcal{C}|} \mathbf{1}_{B_{j}^{\prime}(\mathcal{G})} \frac{p_{j}}{\widetilde{p}_{j}} Y_{Z V R D}\left(\mathcal{G}_{j}\right) \\
& =q_{\mathcal{C}}+\left(\sum_{k=1}^{|\mathcal{C}|} \mathbb{P}\left[B_{k}\right] q\left(\mathcal{G}_{k}\right)\right) \sum_{j=1}^{|\mathcal{C}|} \mathbf{1}_{B_{j}^{\prime}(\mathcal{G})} \frac{1}{q\left(\mathcal{G}_{j}\right)} Y_{Z V R D}\left(\mathcal{G}_{j}\right),
\end{aligned}
$$

the subscript $Z V R D$ being for Zero-Variance $R V R$.

PROPOSITION 5.1. Estimator $Y_{Z V R D}$ has variance $\widetilde{\operatorname{Var}}\left[Y_{Z V R D}\right]=0$, i.e., it always exactly yields the expected value $q(\mathcal{G})$.

PROOF. This can be proved by induction playing with variances, or just by showing that $Y_{Z V R D}$ always gives $q(\mathcal{G})$. From (14), using the fact that

$$
q(\mathcal{G})=q_{\mathcal{C}}+\sum_{k=1}^{|\mathcal{C}|} \mathbb{P}\left[B_{k}\right] q\left(\mathcal{G}_{k}\right)
$$

the result is immediate as soon as $Y_{Z V R D}\left(\mathcal{G}_{j}\right)=q\left(\mathcal{G}_{j}\right) \forall 1 \leq j \leq|\mathcal{C}|$ because $\sum_{j=1}^{|\mathcal{C}|} \mathbf{1}_{B_{j}^{\prime}(\mathcal{G})} Y_{Z V R D}\left(\mathcal{G}_{j}\right) / q\left(\mathcal{G}_{j}\right)$ always gives 1 . This is verified by induction, from the simplest elementary topologies, for which $Y_{Z V R D}$ is replaced by the reliability of the graph.

Unfortunately, implementing this estimator requires the knowledge of the $q\left(\mathcal{G}_{i}\right)$, but if we knew them, there would be no need for simulation. Instead, we suggest to use $\widehat{q}\left(\mathcal{G}_{i}\right)$ as an approximation of the probability $q\left(\mathcal{G}_{i}\right)$, and to plug it into (13). If we can get a valid approximation at a moderate computational cost, the variance of the estimator can be very low. Using this approximation, the estimator $Y_{A Z V R D}$ (subscript $A Z V R D$ being for Approximate Zero-Variance RVR) is expressed as:

$$
Y_{A Z V R D}=q_{\mathcal{C}}+\left(\sum_{k=1}^{|\mathcal{C}|} \mathbb{P}\left[B_{k}\right] \widehat{q}\left(\mathcal{G}_{k}\right)\right) \sum_{j=1}^{|\mathcal{C}|} \mathbf{1}_{B_{j}^{\prime}(\mathcal{G})} \frac{1}{\widehat{q}\left(\mathcal{G}_{j}\right)} Y_{A Z V R D}\left(\mathcal{G}_{j}\right)
$$

We can now prove the following result.

Proposition 5.2. If, at every step of the recursive decomposition algorithm, for all $1 \leq j \leq|\mathcal{C}|$, we have $\widehat{q}\left(\mathcal{G}_{j}\right)=\Theta\left(q\left(\mathcal{G}_{j}\right)\right)$ as $\epsilon \rightarrow 0$, the estimator $Y_{A Z V R D}$ verifies the bounded relative error property.

PROOF. The proof proceeds again by induction and resembles the one for Proposition 4.1. For elementary graphs, $Y_{A Z V R D}$ is just a numerical value (constant), and therefore $\widetilde{\mathbb{E}}\left[Y_{A Z V R D}^{2}\right]=Y_{A Z V R D}^{2}=\widetilde{\mathbb{E}}\left[Y_{A Z V R D}\right]^{2}$ meaning that BRE is obtained. 
The recursion on the second moment is given by

$$
\begin{aligned}
\widetilde{\mathbb{E}}\left[Y_{A Z V R D}^{2}(\mathcal{G})\right]= & q_{\mathcal{C}}^{2}+2 q_{\mathcal{C}} \sum_{j=1}^{|\mathcal{C}|} \mathbb{P}\left[B_{j}\right] \widetilde{\mathbb{E}}\left[Y_{A Z V R D}\left(\mathcal{G}_{j}\right)\right] \\
& +\left(\sum_{k=1}^{|\mathcal{C}|} \mathbb{P}\left[B_{k}\right] \widehat{q}\left(\mathcal{G}_{k}\right)\right) \sum_{j=1}^{|\mathcal{C}|} \frac{\mathbb{P}\left[B_{j}\right] \widetilde{\mathbb{q}}\left(\mathcal{G}_{j}\right)}{\mathbb{E}}\left[Y_{A Z V R D}^{2}\left(\mathcal{G}_{j}\right)\right] .
\end{aligned}
$$

Let $c, d, f_{j}, g_{j}$ and $d_{j}$ be constants such that $q_{\mathcal{C}}=\Theta\left(\epsilon^{c}\right), \widetilde{\mathbb{E}}\left[Y_{A Z V R D}(\mathcal{G})\right]=\Theta\left(\epsilon^{d}\right)$, $\mathbb{P}\left[B_{j}(\mathcal{G})\right]=\Theta\left(\epsilon^{f_{j}}\right), \widehat{q}\left(\mathcal{G}_{j}\right)=\Theta\left(\epsilon^{g_{j}}\right)$ and $\widetilde{\mathbb{E}}\left[Y_{A Z V R D}\left(\mathcal{G}_{j}\right)\right]=\Theta\left(\epsilon^{d_{j}}\right)$. Remember that such constants always exist since the expectations are finite sums of polynomials in $\epsilon$. We have from (15) $d=\min _{j}\left(c, f_{j}+d_{j}\right)$. By induction assumption, $\forall j$, $\widetilde{\mathbb{E}}\left[Y_{A Z V R D}^{2}\left(\mathcal{G}_{j}\right)\right]=\Theta\left(\left(\widetilde{\mathbb{E}}\left[Y_{A Z V R D}\left(\mathcal{G}_{j}\right)\right]\right)^{2}\right)$, so that we get from (16), $\widetilde{\mathbb{E}}\left[Y_{A Z V R D}^{2}(\mathcal{G})\right]=$ $\Theta\left(\epsilon^{\min _{j}\left(2 c, c+f_{j}+d, \min _{k}\left(f_{k}+g_{k}\right)+f_{j}-g_{j}+2 d_{j}\right)}\right)$. But $\widehat{q}\left(\mathcal{G}_{j}\right)=\Theta\left(q\left(\mathcal{G}_{j}\right)\right)$ by assumption with $q\left(\mathcal{G}_{j}\right)=\mathbb{E}\left[Y_{A Z V R D}\left(\mathcal{G}_{j}\right)\right]$, meaning that $g_{j}=d_{j}$. We then have (since the total number of steps is finite)

$$
\widetilde{\mathbb{E}}\left[Y_{A Z V R D}^{2}(\mathcal{G})\right]=\Theta\left(\epsilon^{\min _{j}\left(2 c, c+f_{j}+d, \min _{k}\left(f_{k}+d_{k}\right)+f_{j}+d_{j}\right)}\right) .
$$

One can check that each of the three terms in the minimum are larger than or equal to $2 d$ (with equality at least once). As a consequence,

$$
\widetilde{\mathbb{E}}\left[Y_{A Z V R D}^{2}(\mathcal{G})\right]=\Theta\left(\epsilon^{2 d}\right)=\Theta\left(\left(\widetilde{\mathbb{E}}\left[Y_{A Z V R D}(\mathcal{G})\right]\right)^{2}\right) .
$$

This completes the proof.

Definition 5.3. Define the mincut-maxprob approximation $\widehat{q}(\mathcal{G})$ of $q(\mathcal{G})$ as the maximal probability of a mincut of $\mathcal{G}$. This approximation has the advantage of being relatively simple and easy to compute, in polynomial time.

This mincut-maxprob approximation will be used in the rest of the paper.

PROPOSITION 5.4. With the mincut-maxprob approximation, $\widehat{q}\left(\mathcal{G}_{j}\right)=\Theta\left(q\left(\mathcal{G}_{j}\right)\right)$ as $\epsilon \rightarrow 0$ for all $1 \leq j \leq|\mathcal{C}|$. Therefore, the BRE property is obtained as a consequence of Proposition 5.2.

The proof of this result is provided in [L'Ecuyer et al. 2011]. Intuitively, $q(\mathcal{G})$ is the probability that at least one mincut has all its links failed (more exactly, it is the probability of the union of those events), the leading term in its expansion in $\epsilon$ corresponding to mincuts with maximal probability.

We remark now that with this approximation, not only the BRE property, but also the VRE property can be verified using the mincut-maxprob approximation. The next proposition provides sufficient (but not necessary) conditions with that respect, in a way similar to what has been done in [L'Ecuyer et al. 2011] for IS, when not combined with RVR.

PROPOSITION 5.5. If, at every step of the recursive decompositon algorithm, $\widehat{q}\left(\mathcal{G}_{j}\right)=$ $q\left(\mathcal{G}_{j}\right)+o\left(q\left(\mathcal{G}_{j}\right)\right)$ as $\epsilon \rightarrow 0$ for all $1 \leq j \leq|\mathcal{C}|$, then the VRE property is verified.

In words, if the aproximation corresponds asymptotically to the true value, then we addtionally vanishing relative error.

PROOF. We want to show that

$$
\widetilde{\mathbb{E}}\left[Y_{A Z V R D}^{2}(\mathcal{G})\right]=\left(\widetilde{\mathbb{E}}\left[Y_{A Z V R D}(\mathcal{G})\right]\right)^{2}+o\left(\left(\widetilde{\mathbb{E}}\left[Y_{A Z V R D}(\mathcal{G})\right]\right)^{2}\right)
$$

ACM Transactions on Modeling and Computer Simulation, Vol. V, No. N, Article , Publication date: January YYYY. 
as $\epsilon \rightarrow 0$. The proof again proceeds by induction on the different steps of the recursive decomposition algorithm. Again, computations are exact for elementary graphs, so that the result is true there. Assume now that or all $1 \leq j \leq|\mathcal{C}|, \widetilde{\mathbb{E}}\left[Y_{A Z V R D}^{2}\left(\mathcal{G}_{j}\right)\right]=$ $\left(\widetilde{\mathbb{E}}\left[Y_{A Z V R D}\left(\mathcal{G}_{j}\right)\right]\right)^{2}+o\left(\left(\widetilde{\mathbb{E}}\left[Y_{A Z V R D}\left(\mathcal{G}_{j}\right)\right]\right)^{2}\right)$. Recall that

$$
\left(\widetilde{\mathbb{E}}\left[Y_{A Z V R D}(\mathcal{G})\right]\right)^{2}=\left(q_{\mathcal{C}}+\sum_{j=1}^{|\mathcal{C}|} \mathbb{P}\left[B_{j}\right] \widetilde{\mathbb{E}}\left[Y_{A Z V R D}\left(\mathcal{G}_{j}\right)\right]\right)^{2} .
$$

¿From (16), the result we need to show is that

$$
\begin{aligned}
\left(\sum_{k=1}^{|\mathcal{C}|} \mathbb{P}\left[B_{k}\right] \widehat{q}\left(\mathcal{G}_{k}\right)\right) \sum_{j=1}^{|\mathcal{C}|} \frac{\mathbb{P}\left[B_{j}\right]}{\widehat{q}\left(\mathcal{G}_{j}\right)} \widetilde{\mathbb{E}}\left[Y_{A Z V R D}^{2}\left(\mathcal{G}_{j}\right)\right]= & \left(\sum_{j=1}^{|\mathcal{C}|} \mathbb{P}\left[B_{j}\right] \widetilde{\mathbb{E}}\left[Y_{A Z V R D}\left(\mathcal{G}_{j}\right)\right]\right)^{2} \\
& +o\left(\left(\widetilde{\mathbb{E}}\left[\left(Y_{A Z V R D}(\mathcal{G})\right)^{2}\right) .\right.\right.
\end{aligned}
$$

But

$$
\begin{aligned}
\sum_{k=1}^{|\mathcal{C}|} \mathbb{P}\left[B_{k}\right] \widehat{q}\left(\mathcal{G}_{k}\right) & =\sum_{k=1}^{|\mathcal{C}|} \mathbb{P}\left[B_{k}\right]\left(q\left(\mathcal{G}_{k}\right)+o\left(q\left(\mathcal{G}_{k}\right)\right)\right) \\
& =\sum_{k=1}^{|\mathcal{C}|} \mathbb{P}\left[B_{k}\right] \widetilde{\mathbb{E}}\left[Y_{A Z V R D}\left(\mathcal{G}_{k}\right)\right]+o\left(\widetilde{\mathbb{E}}\left[Y_{A Z V R D}(\mathcal{G})\right]\right)
\end{aligned}
$$

because $q\left(\mathcal{G}_{k}\right)=\widetilde{\mathbb{E}}\left[Y_{A Z V R D}\left(\mathcal{G}_{k}\right)\right]$, and

$$
\begin{aligned}
& \sum_{j=1}^{|\mathcal{C}|} \frac{\mathbb{P}\left[B_{j}\right]}{\widehat{q}\left(\mathcal{G}_{j}\right)} \widetilde{\mathbb{E}}\left[Y_{A Z V R D}^{2}\left(\mathcal{G}_{j}\right)\right]=\sum_{j=1}^{|\mathcal{C}|} \frac{\mathbb{P}\left[B_{j}\right]}{q\left(\mathcal{G}_{j}\right)+o\left(q\left(\mathcal{G}_{j}\right)\right)}\left(\widetilde{\mathbb{E}}\left[\left(Y_{A Z V R D}\left(\mathcal{G}_{j}\right)\right)^{2}\right]+o\left(\widetilde{\mathbb{E}}\left[\left(Y_{A Z V R D}\left(\mathcal{G}_{j}\right)\right)^{2}\right]\right)\right) \\
& =\sum_{j=1}^{|\mathcal{C}|} \frac{\mathbb{\mathbb { E }}\left[B_{j}\right]}{\widetilde{\mathbb{E}}\left[Y_{A Z V R D}\left(\mathcal{G}_{j}\right)\right]+o\left(\widetilde{\mathbb{E}}\left[Y_{A Z V R D}\left(\mathcal{G}_{j}\right)\right]\right)} \times \\
& \left(\left(\widetilde{\mathbb{E}}\left[Y_{A Z V R D}\left(\mathcal{G}_{j}\right)\right]\right)^{2}+o\left(\left(\widetilde{\mathbb{E}}\left[Y_{A Z V R D}\left(\mathcal{G}_{j}\right)\right]\right)^{2}\right)\right) \\
& =\sum_{j=1}^{|\mathcal{C}|} \mathbb{P}\left[B_{j}\right] \widetilde{\mathbb{E}}\left[Y_{A Z V R D}\left(\mathcal{G}_{j}\right)\right]+o\left(\widetilde{\mathbb{E}}\left[Y_{A Z V R D}(\mathcal{G})\right]\right)
\end{aligned}
$$

using the same argument $q\left(\mathcal{G}_{j}\right)=\widetilde{\mathbb{E}}\left[Y_{A Z V R D}\left(\mathcal{G}_{j}\right)\right]$ and the induction assumption, hence the result.

Remark 5.6.

Remark again that the condition provided in the proposition is sufficient and not necessary. It can be made weaker by just considering the assumption at graphs $\mathcal{G}_{j}$ such that $\mathbb{P}\left[B_{j}\right] \widehat{q}\left(\mathcal{G}_{j}\right)=\Theta(q(\mathcal{G}))$, i.e., the subgraphs that contribute to the first order approximation (in terms of $\epsilon$ ) of $q(\mathcal{G})$. But the purpose of our result is to illustrate that a better property than BRE can be obtained, not to provide the weakest possible conditions, that will anyway be hard to verify in practice. 


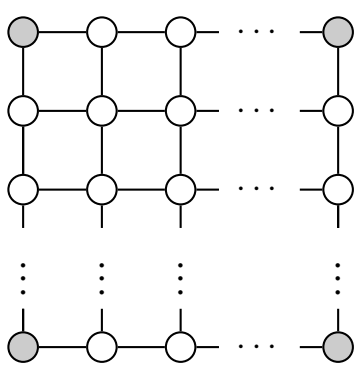

Fig. 4. $G_{k}$ : the Grid Network Topology

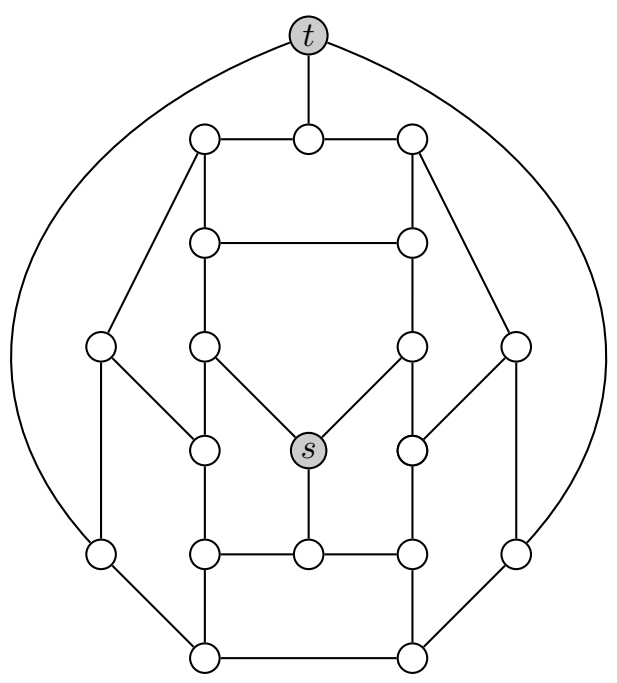

Fig. 5. The "Dodecahedron" topology

\section{NUMERICAL ILLUSTRATIONS}

In this section, we illustrate the behavior of the relative errors of the three estimators presented in this paper. We consider five problems based on topologies taken from previous literature in the subject [Elperin et al. 1991; Hui et al. 2005] :

- The complete networks $C_{6}$ and $C_{10}$. For $C_{10}$, we consider the all-terminal unreliability problem whereas the network $C_{6}$ is exploited in the source-terminal case.

- The grid network $G_{5}$ where the terminals are the four corner nodes (see Figure 4).

- The Dodecahedron topology pictured at Figure 5 where the terminals are the nodes $s$ and $t$.

-A version of Arpanet pictured at Figure 6 where the terminals are the nodes $s$ and $t$.

\subsection{Discussion on the BRE and VRE properties}

Recall that for a fixed sample size $n$, the relative error of a sample mean estimator $\widehat{Y}_{n}$ based on independent samples of r.v. $Y$ with mean $q(\mathcal{G})$ is equal to $R E_{Y}=$ $c_{\alpha} \sqrt{\operatorname{Var}[Y] / n} / q(\mathcal{G})$ where $\operatorname{Var}[Y]$ is the variance of $Y$ (Equation 4). For a fixed sample size $n$, the best mean sample estimator, in terms of accuracy, is the one with the small- 


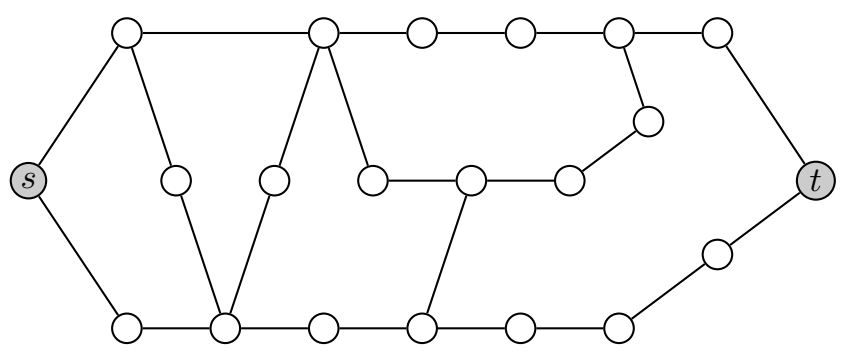

Fig. 6. A version of the Arpanet topology.

Table I. Evolution of the relative errors of SMC, RVR, BRD and AZVRD estimators, as a function of the link unreliability on five networks. All recursive estimators use a mincut of maximal probability at each recursive step.

\begin{tabular}{|c|c|c|c|c|c|c|}
\hline Network & $\epsilon$ & $q(\mathcal{G})$ & $\frac{\sqrt{n} \times R E_{S M C}}{c_{\alpha}}$ & $\frac{\sqrt{n} \times R E_{R V R}}{c_{\alpha}}$ & $\frac{\sqrt{n} \times R E_{B R D}}{c_{\alpha}}$ & $\frac{\sqrt{n} \times R E_{A Z V R D}}{c_{\alpha}}$ \\
\hline Arpanet & $5 \mathrm{e}-01$ & $9.6398994 \mathrm{e}-01$ & $1.93 \mathrm{e}-01$ & $6.33 \mathrm{e}-02$ & $4.16 \mathrm{e}-01$ & $4.27 \mathrm{e}-01$ \\
\hline Arpanet & $3 \mathrm{e}-01$ & $6.8150724 \mathrm{e}-01$ & $6.84 \mathrm{e}-01$ & $3.20 \mathrm{e}-01$ & $1.10 \mathrm{e}+00$ & $1.35 \mathrm{e}+00$ \\
\hline Arpanet & $1 \mathrm{e}-01$ & $9.5422918 \mathrm{e}-02$ & $3.08 \mathrm{e}+00$ & $1.27 \mathrm{e}+00$ & $2.01 \mathrm{e}+00$ & $3.24 \mathrm{e}+00$ \\
\hline Arpanet & $1 \mathrm{e}-03$ & $6.0558106 \mathrm{e}-06$ & $4.06 \mathrm{e}+02$ & $2.09 \mathrm{e}+01$ & $1.24 \mathrm{e}+00$ & $9.67 \mathrm{e}-01$ \\
\hline Arpanet & $1 \mathrm{e}-05$ & $6.0005600 \mathrm{e}-10$ & $4.08 \mathrm{e}+04$ & $2.11 \mathrm{e}+02$ & $1.26 \mathrm{e}+00$ & $9.82 \mathrm{e}-02$ \\
\hline Dod & $5 \mathrm{e}-01$ & $7.0974499 \mathrm{e}-01$ & $6.39 \mathrm{e}-01$ & $1.77 \mathrm{e}-01$ & $9.17 \mathrm{e}-01$ & $5.17 \mathrm{e}-01$ \\
\hline Dod & $3 \mathrm{e}-01$ & $1.6851806 \mathrm{e}-01$ & $2.22 \mathrm{e}+00$ & $5.70 \mathrm{e}-01$ & $1.93 \mathrm{e}+00$ & $7.70 \mathrm{e}-01$ \\
\hline Dod & $1 \mathrm{e}-01$ & $2.8796013 \mathrm{e}-03$ & $1.86 \mathrm{e}+01$ & $8.37 \mathrm{e}-01$ & $9.53 \mathrm{e}-01$ & $2.76 \mathrm{e}-01$ \\
\hline Dod & $1 \mathrm{e}-03$ & $2.0060181 \mathrm{e}-09$ & $2.23 \mathrm{e}+04$ & $7.08 \mathrm{e}-01$ & $7.06 \mathrm{e}-01$ & $1.59 \mathrm{e}-02$ \\
\hline Dod & $1 \mathrm{e}-05$ & $2.0000600 \mathrm{e}-15$ & $2.24 \mathrm{e}+07$ & $7.07 \mathrm{e}-01$ & $7.07 \mathrm{e}-01$ & $1.58 \mathrm{e}-03$ \\
\hline Grid5 & $5 \mathrm{e}-01$ & $9.6062484 \mathrm{e}-01$ & $2.02 \mathrm{e}-01$ & $2.66 \mathrm{e}-02$ & $4.55 \mathrm{e}-01$ & $1.01 \mathrm{e}-01$ \\
\hline Grid5 & $3 e-01$ & $5.2094890 \mathrm{e}-01$ & $9.59 \mathrm{e}-01$ & $1.53 \mathrm{e}-01$ & $1.17 \mathrm{e}+00$ & $2.29 \mathrm{e}-01$ \\
\hline Grid5 & $1 \mathrm{e}-01$ & $4.8160510 \mathrm{e}-02$ & $4.45 \mathrm{e}+00$ & $1.40 \mathrm{e}-01$ & $1.09 \mathrm{e}+00$ & $1.35 \mathrm{e}-01$ \\
\hline Grid5 & $1 \mathrm{e}-03$ & $4.0080020 \mathrm{e}-06$ & $4.99 \mathrm{e}+02$ & $1.58 \mathrm{e}-02$ & $1.14 \mathrm{e}+00$ & $1.37 \mathrm{e}-02$ \\
\hline Grid5 & $1 \mathrm{e}-05$ & $4.0000800 \mathrm{e}-10$ & $5.00 \mathrm{e}+04$ & $1.58 \mathrm{e}-03$ & $1.15 \mathrm{e}+00$ & $1.37 \mathrm{e}-03$ \\
\hline C6 & $5 \mathrm{e}-01$ & $7.6416016 \mathrm{e}-02$ & $3.48 \mathrm{e}+00$ & $1.15 \mathrm{e}-01$ & $3.43 \mathrm{e}-01$ & $1.12 \mathrm{e}-01$ \\
\hline C6 & $3 e-01$ & $5.2672775 \mathrm{e}-03$ & $1.37 \mathrm{e}+01$ & $9.61 \mathrm{e}-02$ & $5.32 \mathrm{e}-01$ & $9.06 \mathrm{e}-02$ \\
\hline C6 & $1 \mathrm{e}-01$ & $2.0076587 \mathrm{e}-05$ & $2.23 \mathrm{e}+02$ & $1.78 \mathrm{e}-02$ & $7.53 \mathrm{e}-01$ & $1.71 \mathrm{e}-02$ \\
\hline C6 & $1 \mathrm{e}-03$ & $2.0000000 \mathrm{e}-15$ & $2.24 \mathrm{e}+07$ & $1.58 \mathrm{e}-05$ & $8.65 \mathrm{e}-01$ & $1.58 \mathrm{e}-05$ \\
\hline C6 & $1 \mathrm{e}-05$ & $2.0000001 \mathrm{e}-25$ & $2.24 e+12$ & $1.89 \mathrm{e}-08$ & $8.66 \mathrm{e}-01$ & $1.89 \mathrm{e}-08$ \\
\hline C10 & $5 \mathrm{e}-01$ & $1.9550825 \mathrm{e}-02$ & $7.08 \mathrm{e}+00$ & $2.10 \mathrm{e}-01$ & $3.65 \mathrm{e}+01$ & $3.13 \mathrm{e}-01$ \\
\hline C10 & $3 e-01$ & $1.9690832 \mathrm{e}-04$ & $7.13 \mathrm{e}+01$ & $2.21 \mathrm{e}-01$ & $7.33 \mathrm{e}+01$ & $4.35 \mathrm{e}-01$ \\
\hline $\mathrm{C} 10$ & $1 \mathrm{e}-01$ & $1.0000004 \mathrm{e}-08$ & $1.00 \mathrm{e}+04$ & $3.33 \mathrm{e}-01$ & $1.04 \mathrm{e}+02$ & $5.95 \mathrm{e}-01$ \\
\hline C10 & $1 \mathrm{e}-03$ & $5.9991786 \mathrm{e}-27$ & $1.29 e+13$ & $5.27 \mathrm{e}+00$ & $1.17 \mathrm{e}+01$ & $4.99 \mathrm{e}-01$ \\
\hline C10 & $1 \mathrm{e}-05$ & $4.1102231 \mathrm{e}-45$ & $1.56 e+22$ & $7.69 \mathrm{e}+01$ & $2.63 \mathrm{e}+00$ & $2.70 \mathrm{e}-01$ \\
\hline
\end{tabular}

est relative error. To compare the accuracy of the methods, we tabulate at columns 5 , 6 and 7 of Table I, the exact values of $\sqrt{n} \times R E_{Y} / c_{\alpha}=\sqrt{\operatorname{Var}\{Y\}} / q(\mathcal{G})$, for the estimators RVR, BRD and AZVRD. Variances needed for these computations are exactly computed by recursive algorithms based on formulas (7), (11) and (15), whereas the exact unreliability $q(\mathcal{G})$ (shown in column 3 ) is computed by an exact algorithm based on the factoring method [Satyarayana and Chang 1983].

Our objective in this subsection is to analyze, for the five different network topologies already described in the previous subsection, how does the relative error of each method evolve when the link unreliability $q_{e}$ (assumed the same for all links) decreases. We can observe that on two of the five networks, the Arpanet and the $C_{10}$ networks, that the basic RVR estimator has a RE which grows with $q_{e}$. This behavior is a confirmation of that RVR does not verify BRE property in general. This does not prevent the RVR estimator from being very efficient on some other topologies though. 
For instance, its RE is almost invariant for small values of $q_{e}$ on the Dodecahedron topology. We can even observe a better behavior on the networks $C_{6}$ and $G_{5}$, where asymptotically the $\mathrm{RE}$ decreases when the rarity increases (a behavior consistent with the Vanishing Relative Error property being satisfied).

We showed theoretically that the two other estimators, BRD and AZVRD, always satisfy the BRE property. As expected, this behavior is observed experimentally on each of the five network considered in Table I. Looking now at the vanishing relative error property, we observe that RVR seems to verify it on the networks $C_{6}$ and $G_{5}$ as evoked above, and that AZVRD actually satisfies it on all considered networks here, with the possible exception of $C_{10}$, where the values seem quite constant and are consistent with BRE. On the other hand, the version BRD does not satisfy VRE property on any of the topologies studied here.

Let us now analyze the accuracy of the three versions when compared to the SMC method as it is usually done in this context. As we have the exact value of $q(\mathcal{G})$, the relative error of the SMC estimator (shown in column 4 of Table I) is obtained exactly from the formula

$$
\sqrt{n} \times R E_{S M C} / c_{\alpha}=\sqrt{q(\mathcal{G})(1-q(\mathcal{G})} / q(\mathcal{G}) .
$$

Comparing columns 4 and 5 of Table I it is possible to see that, as expected, RVR leads to a gain in accuracy on all of the 20 configurations in Table I. It is actually proved in [Cancela and El Khadiri 2003] that the relative error is always reduced with respect to SMC using RVR, whatever the unreliability and the topology. On the other hand, BRD and AZVRD do not always verify this property, and in some cases have errors larger than SMC. Among the considered configurations, this kind of bad behavior is observed when AZVRD is applied to the Arpanet topology with common link unreliability values equal to $0.5,0.3$ or to 0.1 (non rare enough cases). For the method BRD, this problem happens in almost all networks for unreliabilities equal to 0.5 and 0.3 , and in several cases for unreliabilities equal to 0.1 .

\subsection{Estimations of the network unreliability and of the variances of the estimators on some configurations of the complete topology $C_{10}$}

Generally, we do not know either the unreliability parameter $q(\mathcal{G})$ or the variance $V_{M}$ of the estimator related to the method $M$ and we have to estimate them. An estimator of $q(\mathcal{G})$ must offer, in a reasonable CPU execution time, an estimate close to the exact value and its variance also needs to be correctly estimated in order to supply a robust confidence interval.

Let us denote (as defined in Section 2) $\widehat{Y}_{n}$ the sample mean estimator based on the r.v. $Y$ with expectation equal to $q(\mathcal{G})$ and related to the method $M$, and $\widehat{V}_{M}$ an estimator of its variance.

Table II supplies simulation results obtained by the three methods proposed in this paper for the network $C_{10}$, with sample size $n=10^{6}$. The first column of the table shows the applied methods. The second column gives the values of the common link unreliability $q_{e}$, which lies in the set $\{0.5,0.1,0.05,0.01\}$. Column 3 gives the estimations of the all terminal network unreliability measure obtained by the three methods, while column 4 corresponds to the exact unreliability values. In column 6 , we tabulate the associated estimations of the related variances. To measure the quality of these estimations, we take advantage of the fact that we also have exact algorithms leading to the exact values of both $q(\mathcal{G})$ and $\operatorname{Var}\left[\widehat{Y}_{n}\right]$. More precisely, we exploit them to compute the relative deviations observed (expressed in percent form)

$$
\Delta_{\widehat{Y}_{n} / q(\mathcal{G})}(\%)=100 \times\left|\widehat{Y}_{n}-q(\mathcal{G})\right| / q(\mathcal{G})
$$


which helps to analyze the quality of the estimation $\widehat{Y}_{n}$ of $q(\mathcal{G})$, and

$$
\Delta_{\widehat{V}_{M} / \operatorname{Var}\left[\widehat{Y}_{n}\right]}(\%)=100 \times\left|\widehat{V}_{M}-\operatorname{Var}\left[\widehat{Y}_{n}\right]\right| / \operatorname{Var}\left[\widehat{Y}_{n}\right],
$$

which gives the relative deviation observed for the variance estimator. If this last value is high the supplied confidence interval may be of low quality because the confidence interval bounds are estimated with large errors. Execution times are presented in the last column of Table II and correspond to programs prepared following the efficient implementation ideas proposed in [Cancela and El Khadiri 2003], using Microsoft Visual C++ 6.0 language and a machine with Intel Core Duo CPU with frequency equal to $2.5 \mathrm{GHZ}$ and 4 Go of RAM. Results in columns 5, 8 and 9 illustrate that all versions provide accurate estimates in reasonable execution times. The balanced version takes more time to realize the $n$ experiments, which is also observed on all others benchmarks we ran, it is also the method with the highest deviation in the estimation of the variance, which nevertheless attains results within a few percent points of the exact one.

Table II. Measure of the quality of the estimates of the all-terminal network unreliability parameter by the three methods and the measure of the quality of estimating the variances of these estimators. The considered network is $C_{10}$ and the sample size is equal to $10^{6}$. All recursive estimators use a mincut of maximal probability at each recursive step.

\begin{tabular}{|l|c|c|c|c|c|c|c|c|}
\hline & $q$ & $\widehat{Y}_{n}$ & $q(\mathcal{G})$ & $\Delta_{\widehat{Y}_{n} / q(\mathcal{G})}$ & $n \times \widehat{V}_{M}$ & $n \times \operatorname{Var}\left[\widehat{Y}_{n}\right]$ & $\Delta_{\widehat{V}_{M} / \operatorname{Var}\left[\widehat{Y}_{n}\right]}$ & $T(s)$ \\
& & & & $\%$ & & & \\
\hline AZVRD & 0.50 & $1.9548012 \mathrm{e}-02$ & $1.9550825 \mathrm{e}-02$ & 0.01 & $3.7355648 \mathrm{e}-05$ & $3.7353248 \mathrm{e}-05$ & 0.01 \\
BRD & 0.50 & $1.9349264 \mathrm{e}-02$ & $1.9550825 \mathrm{e}-02$ & 1.03 & $4.2528047 \mathrm{e}-01$ & $5.1052587 \mathrm{e}-01$ & 16.70 \\
RVR & 0.50 & $1.9521478 \mathrm{e}-02$ & $1.9550825 \mathrm{e}-02$ & 0.15 & $1.5804583 \mathrm{e}-05$ & $1.6907182 \mathrm{e}-05$ & 6.52 \\
\hline AZVRD & 0.10 & $1.0000068 \mathrm{e}-08$ & $1.0000004 \mathrm{e}-08$ & 0.00 & $3.5429607 \mathrm{e}-17$ & $3.5429417 \mathrm{e}-17$ & 0.00 \\
BRD & 0.10 & $1.0000183 \mathrm{e}-08$ & $1.0000004 \mathrm{e}-08$ & 0.00 & $1.1155417 \mathrm{e}-12$ & $1.0777062 \mathrm{e}-12$ & 3.51 \\
RVR & 0.10 & $9.9993513 \mathrm{e}-09$ & $1.0000004 \mathrm{e}-08$ & 0.01 & $1.1021122 \mathrm{e}-17$ & $1.1121715 \mathrm{e}-17$ & 0.90 \\
\hline AZVRD & 0.05 & $1.9531454 \mathrm{e}-11$ & $1.9531321 \mathrm{e}-11$ & 0.00 & $1.5889951 \mathrm{e}-22$ & $1.5889827 \mathrm{e}-22$ & 0.00 & $<1$ \\
BRD & 0.05 & $1.9913558 \mathrm{e}-11$ & $1.9531321 \mathrm{e}-11$ & 1.96 & $5.0125550 \mathrm{e}-18$ & $4.6312871 \mathrm{e}-18$ & 8.23 \\
RVR & 0.05 & $1.9529890 \mathrm{e}-11$ & $1.9531321 \mathrm{e}-11$ & 0.01 & $7.9375701 \mathrm{e}-23$ & $8.0317529 \mathrm{e}-23$ & 1.17 \\
\hline AZVRD & 0.01 & $1.0230430 \mathrm{e}-17$ & $1.0230678 \mathrm{e}-17$ & 0.00 & $5.2667158 \mathrm{e}-35$ & $5.2729342 \mathrm{e}-35$ & 0.12 & $<$ \\
BRD & 0.01 & $9.9902412 \mathrm{e}-18$ & $1.0230678 \mathrm{e}-17$ & 2.35 & $1.2230244 \mathrm{e}-30$ & $1.3391655 \mathrm{e}-30$ & 8.67 \\
RVR & 0.01 & $1.0229746 \mathrm{e}-17$ & $1.0230678 \mathrm{e}-17$ & 0.01 & $1.0436196 \mathrm{e}-34$ & $1.0563497 \mathrm{e}-34$ & 1.21 & $<1$ \\
\hline
\end{tabular}

\section{CONCLUSIONS AND POSSIBLE IMPROVEMENTS}

To summarize, the main contributions of this paper are:

a) the study of the asymptotic properties of the Recursive Variance Reduction method, showing that RVR does not verify the Bounded Relative Error property in general, although it does for some particular topologies. We have also shown in Section 3 that, on some very simple topologies, the order in which the links of the network are considered can lead to either verifying or not verifying the BRE property.

b) The proposal of two new variance reduction simulation methods, called Balanced Recursive Decomposition, and Approximate Zero Variance Recursive Decomposition. These new methods combine ideas from RVR and from Importance Sampling techniques. We show that BRD verifies the BRE property for all network topologies; and that is also the case of AZVRD, when using a mincut-maxprob approximation to define the importance sampling change of probability distribution. We also give a sufficient condition for AZVRD to verify the Vanishing Relative Error property.

c) Numerical illustrations for different topologies, which show that the properties mentioned are observed empirically, and allow to compare the performance (in terms of precision and computing time) of the three methods. 
As a conclusion, based on BRE/VRE properties discussions and the comparisons with the SMC method, we suggest to use the RVR method when $q_{e}$ is not very small (between 0.5 and 0.1) and AZVRD otherwise as this guarantees BRE will be verified.

Future work includes to further study the impact of the choice of the cut and the order of consideration of the links within the RVR method, as well as trying to develop a method which can always attain a variance reduction over standard Monte Carlo (like RVR does) and at the same time guarantee BRE (like AZVRD does). It is also of interest to study in more detail in which cases the VRE property can be attained, and try to better understand the empirical behavior of RVR, which seems to verify the property, at least for some topologies.

\section{References}

S. Asmussen and P. W. Glynn. 2007. Stochastic Simulation. Springer-Verlag, New York.

Michael O. Ball. 1986. Computational Complexity of Network Reliability Analysis: An Overview. IEEE Transactions on Reliability 35, 3 (Aug. 1986), 230-239. DOI :http://dx.doi.org/10.1109/TR.1986.4335422

H. Cancela and M. El Khadiri. 1995. A recursive variance-reduction algorithm for estimating communication-network reliability. IEEE Transactions on Reliability 44, 4 (1995), 595-602.

H. Cancela and M. El Khadiri. 1996. An Improvement to the Total Hazard Method for System Reliability Simulation. Probability in the Engineering and Informational Sciences 10, 2 (1996), 187-196.

H. Cancela and M. El Khadiri. 1998. Series-Parallel Reductions in Monte Carlo Network Reliability Evaluation. IEEE Trans. Rel. 47, 2 (June 1998), 159-164.

H. Cancela and M. El Khadiri. 2003. The recursive variance-reduction simulation algorithm for network reliability evaluation. IEEE Transactions on Reliability 52, 2 (2003), 207-212.

H. Cancela, M. El Khadiri, and G. Rubino. 2009. Rare events analysis by Monte Carlo techniques in static models. In Rare Event Simulation using Monte Carlo Methods, G. Rubino and B. Tuffin (Eds.). John Wiley \& Sons, 145-170.

H. Cancela, M. El Khadiri, and G.Rubino. 2012. A new simulation method based on the RVR principle for the rare event network reliability problem. Annals of Operations Research 196, 1 (July 2012), 111-136.

T. Elperin, I. Gertsbakh, and M. Lomonosov. 1991. Estimation of Network Reliability Using Graph Evolution Models. IEEE Transactions on Reliability 40, 5 (December 1991).

G. S. Fishman. 1986. A Comparison of Four Monte Carlo Methods for Estimating the Probability of s-t Connectedness. IEEE Transactions on reliability 35, 2 (June 1986), 145-155.

P.W. Glynn, G. Rubino, and B. Tuffin. 2009. Robustness Properties and Confidence Interval Reliability Issues. In Rare Event Simulation using Monte Carlo Methods, G. Rubino and B. Tuffin (Eds.). John Wiley \& Sons, 63-84.

P. Heidelberger, P. Shahabuddin, and V.F. Nicola. 1994. Bounded relative error in estimating transient measures of highly dependable non-Markovian systems. ACM Transactions on Modeling and Computer Simulation 4, 2 (April 1994), 137-164.

K.P. Hui, N.Bean, M. Kraetzl, and D.P. Kroese. 2005. The Cross-Entropy Method for Network Reliability Estimation. Annals of Operations Research 134 (2005), 101-118.

C.H. Jun and S.M. Ross. 1992. System Reliability by Simulation: Random Hazards Versus Importance Sampling. Probability in the Engineering and Informational Sciences 6 (1992).

H. Kumamoto, K. Tanaka, K. Inoue, and E.J. Henley. 1980. Dagger-Sampling Monte Carlo for System Unavailability Evaluation. IEEE Trans. Reliab. R-29, 2 (June 1980), 122-125.

P. L'Ecuyer, J. H. Blanchet, B. Tuffin, and P. W. Glynn. 2010. Asymptotic Robustness of Estimators in RareEvent Simulation. ACM Transactions on Modeling and Computer Simulation 20, 1 (2010), Article 6.

P. L'Ecuyer, G. Rubino, S. Saggadi, and B. Tuffin. 2011. Approximate Zero-Variance Importance Sampling for Static Network Reliability Estimation. IEEE Transactions on Reliability 8, 4 (2011), 590-604.

P. L'Ecuyer and B. Tuffin. 2008. Approximate Zero-Variance Simulation. In Proceedings of the 2008 Winter Simulation Conference. IEEE Press, 170-181.

M. Lomonosov. 1994. On Monte Carlo Estimates in Network Reliability. Probability in the Engineering and Informational Sciences 8 (1994), 245-264.

L Murray, H. Cancela, and G. Rubino. 2013. A Splitting Algorithm for Network Reliability. IIE Transactions 45, 2 (2013), 177-189.

M. K. Nakayama. 1996. General Conditions for Bounded Relative Error in Simulations of Highly Reliable Markovian Systems. Advances in Applied Probability 28 (1996), 687-727. 
S.M. Ross. 1994. A New Simulation Estimator of System Reliability. Journal of Applied Mathematics and Stochastic Analysis 7, 3 (1994).

G. Rubino. 1998. Network reliability evaluation. In State-of-the art in performance modeling and simulation, K. Bagchi and J. Walrand (Eds.). Gordon and Breach Books.

G. Rubino and B. Tuffin (Eds.). 2009. Rare Event Simulation using Monte Carlo Methods. John Wiley \& Sons.

A. Satyarayana and M.K. Chang. 1983. Network Reliability and the Factoring Theorem. Networks 13 (1983), 107-120.

José Villén-Altamirano. 2007. Rare event RESTART simulation of two-stage networks. European Journal of Operations Research 179, 1 (May 2007), 148-159. 